\title{
Impact of model for end-stage liver disease (MELD) score for mortality in patients undergoing coronary bypass surgery
}

\author{
Dolapoglu A ${ }^{1}$, Avci E ${ }^{2}$, Argan $\mathrm{O}^{2}$, Safak $\mathrm{O}^{2}$, Gorcan $\mathrm{A}^{2}$, Akgun $\mathrm{DE}^{2}$, Catalkaya $\mathrm{S}^{3}$, Kiris $\mathrm{T}^{4}$ \\ Department of Cardiovascular Surgery, Balikesir University Medical School, Balikesir Turkey. \\ ahmetdolapoglu@yahoo.com
}

\section{ABSTRACT}

OBJECTIVES: The aim of the study is to evaluate the predictive value of the model for end-stage liver disease (MELD) score for mortality in stable angina pectoris patients undergoing coronary artery bypass graft (CABG) surgery.

METHODS: We retrospectively analyzed 261 consecutive patients with stable angina pectoris who underwent CABG while not being on anticoagulant therapy. The patients were divided into two groups: survivors and non-survivors. The MELD score was calculated for all patients. The all-cause mortality within postoperative 12 months was the primary end point of the study.

RESULTS: The follow-up period was 12 months. The non-survivors were older $(72.0 \pm 6.1$ vs $62.4 \pm 8.4$ $p<0.001)$. The MELD score was significantly higher in the non-survivors group $(7.5 \pm 1.2$ vs $6.7 \pm 0.7$, $p<0.001)$. The MELD score $(p=0.001)$ was an independent predictor of postoperative one-year mortality. The addition of MELD score to EuroSCORE II significantly improved the prognostic performance of the EuroSCORE II (EuroSCORE II vs EuroSCORE II plus MELD score: AUCs: 0.792 vs 0.842).

CONCLUSION: Our research showed that the MELD score could be useful to predict mortality in patients who have stable coronary artery disease, and are undergoing CABG surgery (Tab. 3, Fig. 2, Ref. 25). Text in PDF www.elis.sk

KEY WORDS: MELD score, stable coronary artery disease, coronary artery graft bypass, mortality.

\section{Introduction}

The majority of patients with coronary artery disease (CAD) are patients with stable angina pectoris (SAP). In addition to medical treatment used in the treatment of these patients, revascularization methods such as percutaneous coronary intervention (PCI) or coronary artery bypass graft (CABG) can also be applied (1). The efficacy of revascularization in frame of optimal medical treatments in patients with SAP has been previously evaluated in multiple randomized controlled trials $(2,3)$. Although no significant difference has been found among the treatment options, the main restrictive factor for these studies lied in unrealistic levels of medication compliance and lifestyle modification of patients in the medical treatment arm. Thus, there is considerable doubt over the routine clinical practice because of uncertainty of the results of previous randomized controlled trials. However, recent metaanalyses have shown that the survival is better for patients treated with revascularization $(4,5)$.

${ }^{1}$ Department of Cardiovascular Surgery, Balikesir University Medical School, Balikesir Turkey, ${ }^{2}$ Department of Cardiology, Balikesir University Medical School, Balikesir Turkey, ${ }^{3}$ Cardiology Clinic, Edremit State Hospital, Balikesir, Turkey, and ${ }^{4}$ Department of Cardiology, Izmir Katip Celebi University, Ataturk Training and Research Hospital, Izmir Turkey

Address for correspondence: A. Dolapoglu, MD, Balikesir Universitesi Tip Fakultesi Hastanesi, Balikesir, Turkey, 10145.

Phone: +90505.9252300
Coronary bypass surgery is a good method of revascularization, but in patients with SAP, surgery-related mortality and complications are matters for discussion. For the purpose of predicting the outcome in these patients, several risk-scoring systems have been previously developed based on angiographic and/or clinical parameters. These are the Society of Thoracic Surgeons (STS) Score, the European System for Cardiac Operative Risk Evaluation (EuroSCORE), age, creatinine, and ejection fraction (ACEF) score, and SYNTAX Score (6). The model for end-stage liver disease (MELD) score, including the international normalized ratio (INR), total bilirubin (TB) and serum creatinine ( $\mathrm{sCr}$ ) levels, are mainly used to predict outcomes in patients with liver disease (7). Liver disease has been previously cited as a factor worsening the prognosis in cardiac surgery patients, but the existing operative risk-scoring systems such as STS and EuroSCORE do not include exact parameters for liver dysfunction (8). Kiris et al demonstrated that a high MELD score is associated with longterm mortality in patients with acute coronary syndromes (ACS) after PCI (9). Hsieh WC et al described an association between the MELD score and poor survival in patients undergoing cardiac surgery with liver disease (10).

The relationship between mortality and MELD score has not been previously investigated in patients who were undergoing CABG while having stable CAD and normal liver function. In this study, we investigate the association between MELD score and survival in these patients. We also investigated whether the 
MELD score has an effect on the Euroscore risk, which is one of the most commonly used scoring systems for calculating postopoperative mortality.

\section{Material and methods}

We retrospectively identified 285 consecutive patients with SAP who electively underwent an on-pump CABG surgery during the period from September 2015 to July 2017. The patients were followed for 12 months after their operations. Clinical characteristics, laboratory findings, Euroscore II scores, surgical details and postoperative outcomes were obtained from the patients' charts. Patients who had pre-operative baseline INR, sCr and TB levels were included in the study. Patients with a history of liver cirrhosis $(\mathrm{n}=2)$, previous cardiac surgery $(n=1)$, moderate or severe tricuspid regurgitation, pulmonary hypertension or rightheart failure $(n=3)$, those who had been taking any anticoagulant agents (warfarin, heparin, new oral anti-coagulants and lowmolecular-weight heparin, $n=6$ ), patients in critical preoperative state $(n=2)$, patients who had active endocarditis $(n=1)$, patients with concomitant valve disease $(n=4)$, and patients who underwent urgent CABG (n $=2$ ) were excluded from the study. Consequently, the final study population consisted of 261 patients. According to their mortality at follow-up, the patients were divided into two groups: survivors $(n=246)$ and nonsurvivors $(n=25)$. The study was approved by the local ethics committee (number of ethics committee: 2019/39, date: February 20, 2019).

The biochemical panel, complete blood count and coagulation panel of all patients were analyzed at their admission. All measured INR, sCr and TB levels were measured prior to the initiation of any anticoagulant therapy. The INR collection tubes contained $3.2 \%$ sodium citrate. The blood samples were centrifuged and analyzed within 1 hour of sampling. Siemens Healthcare Diagnostic Products kits and calibrators (Marburg, Germany), Abbott Cell-Dyn 3700 autoanalyzer (Abbott Diagnostic, CA, USA) and HemosILRecombiPlasTin 2G reagents (Instrumentation Laboratory, Bedford, MA, USA) were used for the measurement of biochemical panels, complete blood count and INR levels, respectively. The MELD score was calculated using the previously published formula 11.2 $x(\ln I N R)+0.378 x(\ln T B)+0.957 x(\ln$ creatinine $)+0.643(11)$.

All patients were examined using echocardiography before their surgical procedures. A modified Simpson`s method was used for the assessment of left-ventricular end-diastolic/systolic volumes and ejection fractions.

Tab. 1. Baseline characteristics and laboratory findings in the study population.

\begin{tabular}{|c|c|c|c|}
\hline Variable & $\begin{array}{l}\text { Survivors } \\
(\mathrm{n}=236)\end{array}$ & $\begin{array}{l}\text { Non-survivors } \\
\quad(\mathrm{n}=25)\end{array}$ & $\mathrm{p}$ \\
\hline Age (years) & $62.4 \pm 8.4$ & $72.0 \pm 6.1$ & $<0.001$ \\
\hline Female n $(\%)$ & $60(25)$ & $5(20)$ & 0.551 \\
\hline History of COPD n (\%) & $18(8)$ & $4(16)$ & 0.152 \\
\hline Hypertension $\mathrm{n}(\%)$ & $51(22)$ & $6(24)$ & 0.783 \\
\hline Diabetes mellitus n (\%) & $80(34)$ & $11(44)$ & 0.314 \\
\hline Diabetes mellitus on insulin $\mathrm{n}(\%)$ & $34(14)$ & $4(16)$ & 0.830 \\
\hline Hyperlipidemia n (\%) & $45(19)$ & $4(16)$ & 0.709 \\
\hline Extracardiac arteriopathy n (\%) & $27(11)$ & $3(12)$ & 0.934 \\
\hline LIMA usage $\mathrm{n}(\%)$ & $221(94)$ & $24(96)$ & 0.641 \\
\hline NYHA & & & 0.791 \\
\hline In $(\%)$ & $182(77)$ & $21(84)$ & \\
\hline II n $(\%)$ & $45(19)$ & $3(12)$ & \\
\hline III n (\%) & $7(3)$ & $1(4)$ & \\
\hline IV n $(\%)$ & $2(1)$ & $0(0)$ & \\
\hline Current smoking n (\%) & $52(22)$ & $5(20)$ & 0.786 \\
\hline Previous myocardial infarction n (\%) & $51(22)$ & $13(52)$ & 0.001 \\
\hline LMCA lesion $\mathrm{n}(\%)$ & $20(9)$ & $2(8)$ & 0.935 \\
\hline Preoperative Beta blocker usage n (\%) & $87(37)$ & $8(32)$ & 0.631 \\
\hline Target vessel revascularization n (\%) & $12(5)$ & $15(60)$ & $<0.001$ \\
\hline Myocardial reinfarction $\mathrm{n}(\%)$ & $8(3)$ & $10(40)$ & $<0.001$ \\
\hline Post-operative inotropic usage n (\%) & $39(17)$ & $6(24)$ & 0.347 \\
\hline Revision for bleeding $\mathrm{n}(\%)$ & $6(3)$ & $1(4)$ & 0.668 \\
\hline Post-operational dialysis n (\%) & $5(2)$ & $1(4)$ & 0.551 \\
\hline New stroke n $(\%)$ & $7(3)$ & $3(12)$ & 0.025 \\
\hline Redo-surgery n (\%) & $2(1)$ & $0(0)$ & 0.644 \\
\hline Post-operative arrhythmia n (\%) & $32(14)$ & $3(12)$ & 0.828 \\
\hline \multicolumn{4}{|l|}{ Laboratory findings } \\
\hline $\mathrm{BMI}\left(\mathrm{kg} / \mathrm{m}^{2}\right)$ & $23 \pm 2$ & $23 \pm 2$ & 0.980 \\
\hline $\mathrm{SCr}$ adm $(\mathrm{mg} / \mathrm{dl})$ & $0.86 \pm 0.2$ & $0.95 \pm 0.2$ & 0.014 \\
\hline eGFR $\left(\mathrm{mL} /\right.$ minute $\left./ 1.73 \mathrm{~m}^{2}\right)$ & $100.1 \pm 20.7$ & $78.9 \pm 20.4$ & $<0.001$ \\
\hline INR & $0.9 \pm 0.1$ & $1.0 \pm 0.1$ & $<0.001$ \\
\hline $\mathrm{AST}^{*}(\mathrm{U} / \mathrm{L})$ & $23(17-31)$ & $27(21-35)$ & 0.306 \\
\hline $\mathrm{ALT}^{*}(\mathrm{U} / \mathrm{L})$ & $17(10-23)$ & $17(14-21)$ & 0.535 \\
\hline Total bilirubin (mg/dl) & $0.53 \pm 0.17$ & $0.68 \pm 0.16$ & $<0.001$ \\
\hline Serum albumin (mg/dl) & $3.8 \pm 0.5$ & $3.5 \pm 0.3$ & 0.001 \\
\hline WBC $(\times 103 / \mu \mathrm{L})$ & $8.5 \pm 1.9$ & $8.9 \pm 2.4$ & 0.385 \\
\hline Hemoglobin $(\mathrm{g} / \mathrm{dl})$ & $12.8 \pm 1.6$ & $12.5 \pm 1.6$ & 0.432 \\
\hline LVEF (\%) & $50.8 \pm 5.4$ & $48.9 \pm 6.2$ & 0.100 \\
\hline CRP*(mg/dl) & $4(2-5)$ & $3(2-5)$ & 0.649 \\
\hline $\mathrm{CPB}$ time () & $60 \pm 11$ & $65 \pm 12$ & 0.026 \\
\hline Cross-clamp time () & $40 \pm 10$ & $44 \pm 11$ & 0.047 \\
\hline MELD score & $6.7 \pm 0.7$ & $7.5 \pm 1.2$ & $<0.001$ \\
\hline Euro score II & $0.96 \pm 0.5$ & $1.6 \pm 0.8$ & $<0.001$ \\
\hline
\end{tabular}

COPD - chronic obstructive pulmonary disease, LMCA - left main coronary artery.

LVEF - left ventricular ejection fraction, $\mathrm{SCr}$ - serum creatinine at admission, WBC - white blood cell, eGFR estimated glomerular filtration rate, $\mathrm{BMI}$ - body mass index, $\mathrm{CRP}$ - c-reactive protein, $\mathrm{CPB}$ - cardiopulmonary bypass, INR - international normalized ratio, MELD - model for end-stage liver disease.

* Comparison was made using Mann-Whitney $\mathrm{U}$ test at $\mathrm{p}<0.05$, and these values were described by median with inter-quartile range ( 25 th and 75 th percentile). 
SAP was defined as chest pain or discomfort during exercise that is relieved by resting. Left-main CAD was defined as an angiographic narrowing greater than $50 \%$. Anything that causes death was considered to be the cause of death. Therefore, all-cause mortality was defined as any cause of death. New stroke was defined as a newly occurring neurological deficit with radiological confirmation.

All CABG procedures were performed on-pump, with cross-clamped aorta. After general anesthesia, a median sternotomy was performed. Cardiopulmonary bypass was initiated after heparin administration at the proper level of activated clotting time. To stop the heart and procure myocardial protection, cold blood cardioplegia was conducted with multiple doses through antegrade and retrograde ways of administration. While the left internal mammary artery was used to graft the left anterior descending artery as much as possible, the great saphenous vein was used as a graft for all other coronary vessels. The complete revascularization was achieved in all patients.

During 12 post-operative months, all patients were followed for all-cause deaths. The data were obtained from hospital records or by phone interviews with patients and their families.

Statistical tests were performed with SPSS version 16 (SPSS Inc., Chicago, IL, USA). Continuous variables were presented as mean \pm standard deviation, and categorical variables were shown as number of subjects and percentage of their total number. Either the Student's t-test or the Mann-Whitney U test was used to compare parametric values between the two groups, as appropriate. The Chi-squared test was used to compare categorical variables. The Kaplan-Meier method was used to obtain the cumulative survival for total mortality. Significant differences in the survival curves were shown by the A log-rank test. A multivariate Cox regression analysis was used to identify independent predictors for all-cause mortality. The factors that entered the multivariate model included those with p-values $<0.1$ from the univariate analysis and variables with known prognostic values. The predictive values of MELD score, EuroSCORE II, and EuroSCORE II plus MELD score were estimated by the areas under the receiver operating characteristic curve. We used the DeLong test to compare to the MELD score. the area under the curve (AUC) with each of these parameters (12). Moreover, the increased discriminative value of the MELD was also estimated using net reclassification improvement (NRI) and

Tab. 2. Baseline characteristics and laboratory findings in the study population according

\begin{tabular}{|c|c|c|c|}
\hline Variable & $\begin{array}{c}\text { Low MELD score } \\
(\mathrm{n}=205)\end{array}$ & $\begin{array}{l}\text { High MELD score } \\
(\mathrm{n}=56)\end{array}$ & $\mathrm{p}$ \\
\hline Age (years) & $62.9 \pm 8.7$ & $64.7 \pm 8.7$ & 0.173 \\
\hline Female n (\%) & $55(27)$ & $10(18)$ & 0.169 \\
\hline History of COPD n (\%) & $12(6)$ & $10(18)$ & 0.004 \\
\hline Hypertension n (\%) & $41(20)$ & $16(29)$ & 0.169 \\
\hline Diabetes mellitus n (\%) & $70(34)$ & $21(38)$ & 0.641 \\
\hline Diabetes mellitus on insulin $\mathrm{n}(\%)$ & $31(15)$ & $7(13)$ & 0.622 \\
\hline Hyperlipidemia n (\%) & $41(20)$ & $8(14)$ & 0.332 \\
\hline Extracardiac arteriopathy n (\%) & $23(11)$ & $7(12)$ & 0.944 \\
\hline LIMA usage $\mathrm{n}(\%)$ & $192(94)$ & $53(95)$ & 0.786 \\
\hline NYHA & & & 0.655 \\
\hline I n $(\%)$ & $158(77)$ & $45(80)$ & \\
\hline II n $(\%)$ & $39(19)$ & $9(16)$ & \\
\hline III n $(\%)$ & $7(3)$ & $1(2)$ & \\
\hline IV n $(\%)$ & $1(1)$ & $1(2)$ & \\
\hline Current smoking n (\%) & $48(22)$ & $9(20)$ & 0.843 \\
\hline Previous myocardial infarction $\mathrm{n}(\%)$ & $46(22)$ & $18(32)$ & 0.135 \\
\hline LMCA lesion $\mathrm{n}(\%)$ & $16(8)$ & $6(11)$ & 0.487 \\
\hline Preoperative Beta blocker usage n (\%) & $75(37)$ & $20(36)$ & 0.904 \\
\hline Target vessel revascularization $\mathrm{n}(\%)$ & $12(5)$ & $15(60)$ & $<0.001$ \\
\hline Myocardial reinfarction $\mathrm{n}(\%)$ & $10(5)$ & $8(14)$ & 0.014 \\
\hline Post-operative inotropic usage $\mathrm{n}(\%)$ & $32(16)$ & $13(23)$ & 0.182 \\
\hline Revision for bleeding $\mathrm{n}(\%)$ & $6(3)$ & $1(2)$ & 0.639 \\
\hline Post-op dialysis $\mathrm{n}(\%)$ & $4(2)$ & $2(4)$ & 0.473 \\
\hline New stroke $\mathrm{n}(\%)$ & $6(3)$ & $4(7)$ & 0.145 \\
\hline Redo-surgery n (\%) & $2(1)$ & $0(0)$ & 0.458 \\
\hline Post-operative arrhythmia n (\%) & $27(13)$ & $8(14)$ & 0.828 \\
\hline Mortality & $10(5)$ & $15(27)$ & $<0.001$ \\
\hline \multicolumn{4}{|l|}{ Laboratory findings } \\
\hline $\mathrm{BMI}\left(\mathrm{kg} / \mathrm{m}^{2}\right)$ & $23 \pm 2$ & $23 \pm 2$ & 0.764 \\
\hline $\mathrm{SCr}$ adm $(\mathrm{mg} / \mathrm{dl})$ & $0.82 \pm 0.1$ & $1.0 \pm 0.2$ & $<0.001$ \\
\hline eGFR $\left(\mathrm{mL} /\right.$ minute $\left./ 1.73 \mathrm{~m}^{2}\right)$ & $102.5 \pm 19.8$ & $81.8 \pm 20.0$ & $<0.001$ \\
\hline INR & $0.87 \pm 0.08$ & $1.01 \pm 0.12$ & $<0.001$ \\
\hline $\mathrm{AST}^{*}(\mathrm{U} / \mathrm{L})$ & $22(16.5-31)$ & $27.5(21-33)$ & 0.051 \\
\hline ALT*(U/L) & $17(10.5-22.0)$ & $17(11-23.75)$ & 0.923 \\
\hline Total bilirubin (mg/dl) & $0.52 \pm 0.16$ & $0.63 \pm 0.20$ & $<0.001$ \\
\hline Serum albumin (mg/dl) & $3.9 \pm 0.5$ & $3.7 \pm 0.5$ & 0.006 \\
\hline $\mathrm{WBC}\left(\times 10^{3} / \mu \mathrm{L}\right)$ & $8.5 \pm 1.9$ & $8.6 \pm 2.3$ & 0.841 \\
\hline Hemoglobin $(\mathrm{g} / \mathrm{dl})$ & $12.8 \pm 1.6$ & $12.7 \pm 1.8$ & 0.712 \\
\hline LVEF (\%) & $50.7 \pm 5.4$ & $50.2 \pm 5.8$ & 0.520 \\
\hline CRP*(mg/dl) & $4(2-5)$ & $3(2-5)$ & 0.612 \\
\hline CPB time () & $60 \pm 11$ & $63 \pm 12$ & 0.064 \\
\hline Cross-clamp time () & $40 \pm 9$ & $42 \pm 12$ & 0.114 \\
\hline MELD score & $6.4 \pm 0.03$ & $7.87 \pm 1.0$ & $<0.001$ \\
\hline Euro score II & $0.95 \pm 0.45$ & $1.24 \pm 0.83$ & 0.001 \\
\hline
\end{tabular}

COPD - chronic obstructive pulmonary disease, LIMA - Left internal mammarian artery, LMCA - left main coronary artery, NYHA - New York heart association, ALT - alanine aminotransferase, AST - aspartate aminotransferase BMI - body mass index, CPB - cardiopulmonary bypass, CRP - c-reactive protein, eGFR - estimated glomerular filtration rate, EuroSCORE - European System for Cardiac Operative Risk Evaluation, INR - international normalized ratio, LVEF; left ventricular ejection fraction, MELD - model for end-stage liver disease, $\mathrm{SCr}$; serum creatinine at admission, WBC - white blood cell

* Comparison was made using Mann-Whitney U test at $\mathrm{p}<0.05$, and these values were described by median with inter-quartile range ( 25 th and 75 th percentile) 


\section{5-131}

Tab. 3. Univariate and multivariate cox regression analysis for one-year mortality.

\begin{tabular}{|c|c|c|c|c|}
\hline \multirow{2}{*}{ Variables } & \multicolumn{2}{|c|}{ Univariate } & \multicolumn{2}{|c|}{ Multivariate } \\
\hline & $\mathrm{HR}(95 \% \mathrm{CI})$ & $\mathrm{p}$ & HR $(95 \% \mathrm{CI})$ & $\mathrm{p}$ \\
\hline Age* (year) & $1.136(1.079-1.196)$ & $<0.001$ & & \\
\hline $\mathrm{INR} \infty$ & $13.941(5.230-37.164)$ & $<0.001$ & & \\
\hline Total bilirubin * & $24.799(3.994-153.974)$ & 0.001 & & \\
\hline CBP time $\mathbb{R}$ & $1.044(1.011-1.077)$ & 0.009 & $1.080(1.043-1.118)$ & $<0.001$ \\
\hline Cross-clamp time $\AA$ & $1.046(1.009-1.083)$ & 0.013 & & \\
\hline Albumin levels (mg/dl) & $0.340(0.143-0.810)$ & 0.015 & & \\
\hline Admission creatinine levels $(\mathrm{mg} / \mathrm{dl}) *$ & $11.690(1.478-92.465)$ & 0.020 & & \\
\hline MELD score & $2.052(1.555-2.710)$ & $<0.001$ & $2.469(1.453-4.194)$ & 0.001 \\
\hline Previous myocardial infarction * & $3.192(1.456-6.998)$ & 0.004 & & \\
\hline New-stroke & $4.781(1.428-16.001)$ & 0.011 & $19.317(4.383-85.142)$ & $<0.001$ \\
\hline Euro score II & $2.375(1.689-3.338)$ & $<0.001$ & & \\
\hline Target vessel revascularization & $12.510(5.619-27.854)$ & $<0.001$ & $20.343(7.370-56.154)$ & $<0.001$ \\
\hline
\end{tabular}

INR - international normalized ratio, MELD - model fore nd-stage liverdisease, CBP time - cardiopulmonary bypass, EuroSCORE - European System for Cardiac Operative Risk Evaluation.

*These parameters are included in Euro score 2, they are not entered into the multivariate analysis.

$\infty$ These parameters are included in MELD score, they are not entered into the multivariate analysis.

(T) These parameters are included in multivariate analysis separately in order to prevent multicollinearity.

integrated discrimination improvement (13). Two-sided p-values $<0.05$ were considered statistically significant.

\section{Results}

Of the 261 patients, $196(75.1 \%)$ were males and 65 (24.9\%) were females. Tables 1 and 2 show the demographics, clinical

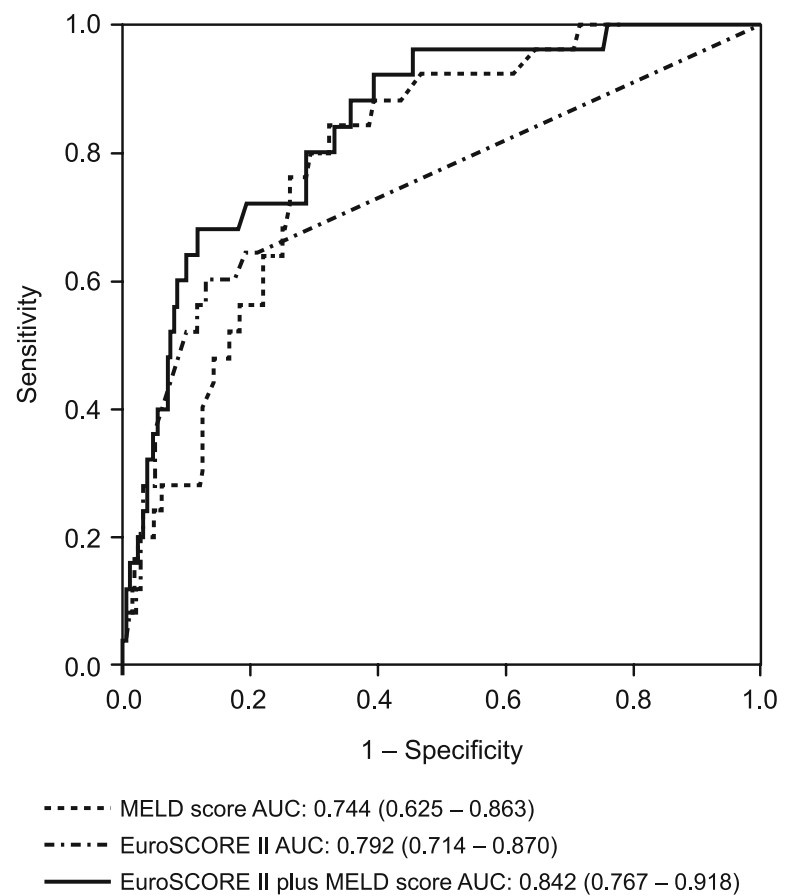

Fig. 1. Receiver operating characteristic (ROC) curves for the MELD score, EuroSCORE II, and EuroSCORE II plus MELD score for predicting 1-year mortality. characteristics and laboratory findings of the population according to survival (survivor/non-survivor groups) and MELD scores (Low/High score groups). Non-survivors were older (72.0 $\pm 6.1 \mathrm{vs}$ $62.5 \pm 8.4 \mathrm{p}<0.001)$. The history of previous myocardial infarction, target vessel revascularization, and new stroke were seen more frequently in non-survivors than in survivors. While $\mathrm{sCr}$, INR and TB were significantly higher in the non-survival group $(0.95 \pm 0.2$ vs $0.86 \pm 0.2, \mathrm{p}=0.014 ; 1.0 \pm 0.1$ vs $0.9 \pm 0.1, \mathrm{p}<0.001$; $0.68 \pm 0.16$ vs $0.53 \pm 0.17, \mathrm{p}<0.001$, respectively), serum albumin levels were significantly lower in this group $(3.5 \pm 0.3 \mathrm{vs}$ $3.8 \pm 0.5, \mathrm{p}=0.001)$. The MELD score was significantly higher

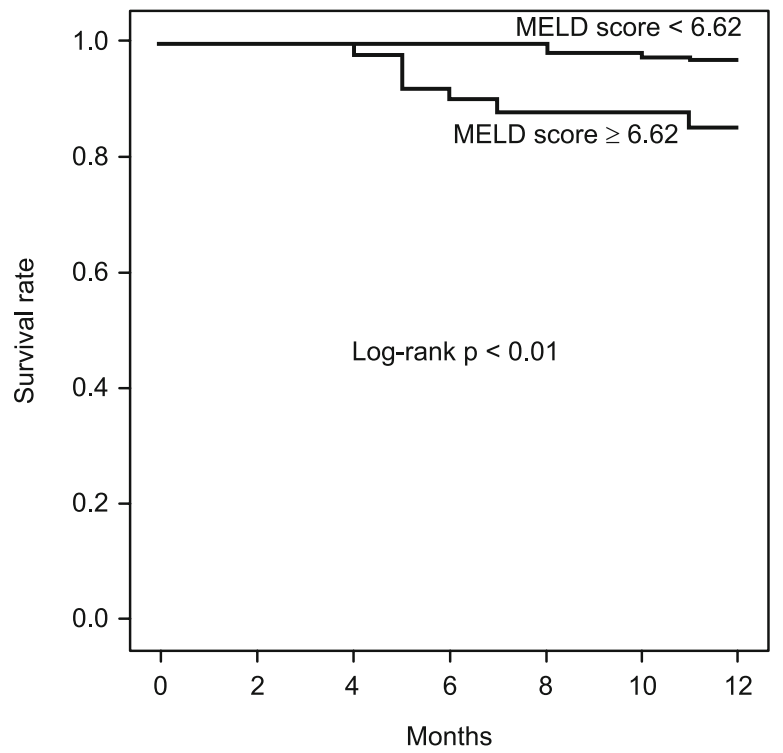

Fig. 2. Kaplan-Meier survival curves of patients according to the MELD score. 
in the non-survival group $(7.5 \pm 1.2$ vs $6.7 \pm 0.7, \mathrm{p}<0.001)$ Also, non-survivors had a high EuroSCORE II compared with survivors $(1.6 \pm 0.8$ vs $0.96 \pm 0.5, \mathrm{p}<0.001)$. For the operative variables, CPB and cross-clamp times were longer in the non-survival group $(65 \pm 12$ vs $60 \pm 11, \mathrm{p}=0.027 ; 44 \pm 11$ vs $40 \pm 10, \mathrm{p}=0.047$, respectively).

The independent predictors for one-year mortality determined with the multivariate cox regression analysis are shown in Tables 3. The MELD score (HR: 2.469, $95 \%$ CI:1.453-4.194, $\mathrm{p}=0.001$ ), new stroke (HR:19.317, $95 \%$ CI: 4.383-85.142, p<0.001), target vessel revascularization (HR: 20.343, $95 \% \mathrm{CI}: 7.370-56.154$, $\mathrm{p}<0.001$ ), and CBP time (HR:1.080, $95 \% \mathrm{CI}: 1.043-1.118$, $\mathrm{p}<0.001$ ) were independently predictive for one-year mortality.

As shown in Figure 1, the area under the curve for the MELD score to predict one-year mortality was 0.744 (95\% CI: $0.625-$ $0.863, \mathrm{p}<0.001)$. For mortality, the AUC of EuroSCORE II was 0.792 (95 \% CI: 0.714-0.870, $\mathrm{p}<0.001)$. When the MELD score was added to EuroSCORE II, the AUC was $0.842(95 \% \mathrm{CI}$ : 0.767-0.918, $z=2.017$, difference $p=0.0437)$. Moreover, the addition of the MELD score to EuroSCORE II was associated with a significant net reclassification improvement estimated at $74.3 \%$ (events: $4 \%$, non-events: $70.3 \%, z=3.534, p=0.0004$ ) and integrated discrimination improvement of $2.81(\mathrm{z}=2.0206$, $\mathrm{p}=0.0433$ ).

A MELD score of 6.62 was identified as the optimal cut-off value with the sensitivity of $64 \%$, specificity of $80.9 \%$, positive predictive value of $26.2 \%$, and negative predictive value of $95.6 \%$ for predicitng 1-year mortality. Patients with MELD score $\geq 6.62$ had significantly lower 1-year survival rate compared with those with MELD score $<6.62$ (log-rank $\mathrm{p}<0.01)$ (Fig. 2).

\section{Discussion}

This study showed that higher MELD scores are associated with increased all-cause mortality in SAP patients treated with CABG surgery. To the best of our knowledge, this is the first study investigating the relationship of MELD scores to the mortality in these patients.

Revascularization significantly decreases mortality and myocardial infarction in high-risk patients with ACS (with or without ST-segment elevation), and it gives the best results. However, the advantages of revascularization have been questioned for the patients with stable coronary artery disease. Even though CAD has decreased by $40 \%$ in developed countries in the last two decades, it is still the major cause of mortality (14). While almost half of this decrease is connected with medical treatment and revascularization, the remaining portion is a function of prevention and reduction of major risk factors. Research by others has indicated that the proportion of patients who are free from angina after revascularization is higher than that of patients provided with conservative medical treatment (15). Based on observations in the literature that the adaptation rate of medical therapies in developed countries is approximately $50 \%$, in a patient with stable angina, the revascularization is an important complement to medical therapy.
Bilirubin, regardless whether conjugated, unconjugated, free or bound with albumin, is known as a potent antioxidant. Bilirubin can protect low-density lipoprotein from oxidation, which is an important phase in the formation of atherosclerosis. A previously published study in patients with SAP indicated that there was an independent, but inverse, association between serum albumin levels and severity of CAD (16). Schwertner et al noted that a reduced serum bilirubin level was directly associated with increased incidence of CAD (17). In a similar study, Novotny et al stated that serum bilirubin is a negative risk factor for CAD (18). In addition, cardiovascular disease is seen at a lower rate in idiopathic unconjugated hyperbilirubinemia, or Gilbert's syndrome which is a congenital disease due to a mutation. All the reviewed studies were conducted in stable patients, but not in a situation of acute stress. Serum bilirubin levels increase during acute stress as a consequence of hemeoxygenase (HO)-I enzyme activity. In addition, there is a positive correlation between serum HO-1 levels and TB levels in patients with ACS. A previous study reported that a high level of serum bilirubin was independently associated with a worse in-hospital outcome in patients with ACS after primary PCI (19). Another study showed associations of the TB level with the development of coronary no-reflow and poor in-hospital outcomes after PCI (20). The study noted that serum bilirubin levels are independently associated with the development of no-reflow and negative cardiac conditions in in-hospital patients with ST segment elevation MI undergoing PCI. Yet, no distinctive relationship was found between TB levels and longterm mortality. Conversely, Kawaguchi et al found a relationship between TB levels and mortality in patients who underwent heart transplantation (21). Similarly, our study demonstrated that TB levels were significantly higher in non-survivors than in survivors, and TB was also an independent predictor of all-cause mortality at follow-up.

A high level of the INR value without anticoagulant medication correlated with six-month mortality in patients with acute pulmonary embolism. An INR level of 1.2 and above was an independent risk factor for mortality among the corresponding patients. Okada et al stated that a higher level of INR was an independent risk factor for all-cause mortality in patients with acute heart failure (22). In a related study, an INR level higher than 1.05 was significantly connected with mortality. An elevated INR level was also an independent risk factor for mortality in our patient population who did not receive anticoagulant therapy. An elevated INR level may be connected with the activation of the coagulation system, inflammatory reactions, neurohumoral activation and hepatic failure.

The sCr level has an important prognostic factor in patients undergoing a cardiopulmonary bypass surgery. Previous studies support our results that the level of basal $\mathrm{sCr}$ in patients who underwent bypass surgery was connected with higher mortality (23). Various factors causing impaired renal function may lead to negative outcomes for this group of patients. These factors include insulin resistance, variance in the extracellular matrix, oxidative stress, inflammatory reactions and endothelial dysfunction, as well as activation of the renin-angiotensin-aldosterone system. 
Since all these parameters depend on accelerated atherosclerosis and endothelial dysfunction, they cause a negative prognosis in patients who underwent bypass surgery.

The MELD score was identified with serum TB, INR and creatinine concentrations (11). This score was primarily developed to evaluate the short-term prognosis in cirrhotic patients who underwent transjugular intrahepatic porto-systemic shunts. Later on, it was used to determine mortality in liver diseases regardless whether etiology was considered, or not. The use of this score also showed excellent performance over the primary allocation of organ in liver transplantations conducted in the United States. It was also noted that the MELD score has predictive significance in determining the complications in consequence of some gastrointestinal, abdominal, bariatric, musculoskeletal, cardiovascular and urologic operations. In these studies, a higher MELD score was an independent variable for some complications such as postoperative wound infection, pulmonary and cardiac complications, and urinary tract infections. These surgical procedures have a high risk of morbidity and mortality in patients with liver failure. Many studies in the literature showed that the postoperative mortality rate in patients having liver dysfunction is $5-85 \%$, depending on the MELD score and type of operation $(24,25)$. The MELD score has been used for risk evaluation also in patients out of surgery. For instance, this score has effectively been used to evaluate the risk potential of diseases such as congestive heart failure and soft tissue infection. At present, the most widely used surgical risk scoring systems such as EuroSCORE do not include any variable for liver function.

In this retrospective study, we found that the MELD score, a scoring method created for the assessment of liver dysfunction, was associated with all-cause mortality in patients with stable CAD who underwent $\mathrm{CABG}$ surgery. According to our results, the MELD score may be used as a successful risk evaluating system for these populations, and the incorporation of the MELD score within STS and EuroSCORE scoring system may provide better prognostic results in the future provided that more prospective and multicenter studies support our findings.

We had some limitations in our study. This study had a retrospective design with a small sample size at a single center. Unfortunately, the current literature does not provide much evidence for coronary bypass surgery with the MELD scoring system. Our study was aimed at meeting the requirement of evaluating predictive risk scoring systems after CABG surgery in patients with SAP and sharing these preliminary results to contribute to future research.

\section{Conclusion}

We have found that the MELD score may be useful in predicting mortality in patients who are undergoing $\mathrm{CABG}$ surgery while having stable coronary artery disease.

\section{References}

1. Knuuti J, Wijns W, Saraste A et al. ESC Scientific Document Group. 2019 ESC Guidelines for the diagnosis and management of chronic coronary syndromes. Eur Heart J 2019; 31. pii: ehz425. doi: 10.1093/eurheartj/ehz425.

2. Boden WE, O'Rourke RA, Teo KK et al. Optimal medical therapy with or without PCI for stable coronary disease. N Engl J Med 2007; 356: $1503-1516$.

3. Trikalinos TA, Alsheikh-Ali AA, Tatsioni A, Nallamothu BK, Kent DM. Percutaneous coronary interventions for non-acute coronary artery disease: a quantitative 20 -year synopsis and a network meta-analysis. Lancet 2009; 373: 911-918.

4. Windecker S, Stortecky S, Stefanini GG et al. Revascularisation versus medical treatment in patients with stable coronary artery disease: network meta-analysis. BMJ 2014; 348: g3859.

5. Mancini GB, Farkouh ME, Brooks MM et al. Medical treatment and revascularization options in patients with type 2 diabetes and coronary disease. J Am Coll Cardiol 2016; 68: 985-995.

6. Ranucci M, Castelvecchio S, Menicanti L, Frigiola A, Pelissero G. Risk of assessing mortality risk in elective cardiac operations: age, creatinine, ejection fraction, and the law of parsimony. Circulation 2009; 119: 3053-3061.

7. Dunn W, Jamil LH, Brown LS et al. MELD accurately predicts mortality in patients with alcoholic hepatitis. Hepatology 2005; 41: 353-358.

8. Lin CH, Lin FY, Wang SS, Yu HY, Hsu RB. Cardiac surgery in patients with liver cirrhosis. Ann Thorac Surg 2005; 79: 1551-1554.

9. Kiris T, Avci E, Çelik A. Combined value of left ventricular ejection fraction and the Model for End-Stage Liver Disease (MELD) score for predicting mortality in patients with acute coronary syndrome who were undergoing percutaneous coronary intervention. BMC Cardiovasc Disord 2018; 18: 44.

10. Hsieh WC, Chen PC, Corciova FC, Tinica G. Liver dysfunction as an important predicting risk factor in patients undergoing cardiac surgery: a systematic review and meta-analysis. Int J Clin Exp Med 2015; 8: 20712-20721.

11. Kamath PS, Wiesner RH, Malinchoc M et al. A model to predict survival in patients with end-stage liver disease. Hepatology 2001; 33 : 464-470.

12. DeLong ER, DeLong DM, Clarke-Pearson DL. Comparing the areas under two or morecorrelated receiver operating characteristic curves: a nonparametric approach. Biometrics 1988; 44: 837-845.

13. Pencina MJ, D’Agostino RB Sr, Steyerberg EW. Extensions of net reclassification improvement calculations to measure usefulness of new biomarkers. Stat Med 2011; 30: 11-21.

14. Lloyd-Jones D, Adams R, Carnethon $M$ et al. Heart disease and stroke statistics: 2009 update: a report from the American Heart Association Statistics Committee and Stroke Statistics Subcommittee. Circulation 2009; 119: 480-486.

15. Simoons ML, Windecker S. Controversies in cardiovascular medicine: chronic stable coronary artery disease: drugs vs. revascularization. Eur Heart J 2010; 31: 530-541.

16. Turfan M, Duran M, Poyraz F et al. Inverse relationship between serum total bilirubin levels and severity of disease in patients with stable coronary artery disease. Coron Artery Dis 2013; 24: 29-32. 
17. Schwertner HA, Jackson WG, Tolan G. Association of low serum concentration of bilirubin with increased risk of coronary artery disease. Clin Chem 1994; 40: 18-23.

18. Novonty L, Vitek L. Inverse relationship between serum bilirubin and atherosclerosis in men: a meta-analysis of published studies. Exp Biol Med (Maywood) 2003; 228: 568-571.

19. Gul M, Uyarel H, Ergelen M et al. Prognostic value of total bilirubin in patients with ST-segment elevation acute myocardial infarction undergoing primary coronary intervention. Am J Cardiol 2013; 111: 166-171.

20. Celik T, Kaya MG, Akpek M et al. Does serum bilirubin level on admission predict TIMI flow grade and in-hospital MACE in patients with STEMI undergoing primary PCI. Angiology 2014; 65: 198-204.

21. Kawaguchi AT, Cabrol C, Gandjbackhch I, Pavie A, Bors V, Muneretto C. Preoperative risk analysis in patients receiving Jarvik-7 artificial heart as bridge to transplantation. Eur J Cardiothorac Surg 1991; 5: 509-514.
22. Okada A, Sugano Y, Nagai T et al. Prognostic value of prothrombin time international normolized ratio in acute decompensated heart failureA combined marker of hepatic insufficiency and hemostatic abnormality. Circ J 2016; 80: 913-923.

23. Litmathe J, Kurt M, Feindt P, Gams E, Boeken U. The impact of pre- and postoperative renal dysfunction on outcome of patients undergoing coronary bypass grafting (CABG). Thorac Cardiovasc Surg 2009; 57: $460-463$

24. Millwala F, Nguyen GC, Thuluvath PJ. Outcomes of patients with cirrhosis undergoing non-hepatic surgery: risk assessment and management. World J Gastroenterol 2007; 13: 4056-4063.

25. Lange EO, Jensen CC, Melton GB, Madoff RD, Kwaan MR. Relationship between Model for End-Stage Liver Disease score and 30-day outcomes for patients undergoing elective colorectal resections: an American College of Surgeons-National Surgical Quality Improvement Program study. Dis Colon Rectum 2015; 58: 494-501.

Received May 25, 2020. Accepted July 15, 2020. 